\title{
On absolute generalized Hausdorff summability of orthogonal series
}

\author{
Xhevat Z. Krasniqi
}




\title{
ON ABSOLUTE GENERALIZED HAUSDORFF SUMMABILITY OF ORTHOGONAL SERIES
}

\author{
XHEVAT Z. KRASNIQI
}

Received 21 February, 2012

\begin{abstract}
In this paper we prove two theorems on absolute generalized Hausdorff summability of orthogonal series. These theorems give some sufficient conditions in terms of coefficients of an orthogonal series under which it is absolute generalized Hausdorff summable. In addition, it is verified that several known results are corollaries of the new results.
\end{abstract}

2010 Mathematics Subject Classification: 42C15; 42C20; 40F05

Keywords: orthogonal series, absolute summability, Hausdorff matrices

\section{INTRODUCTION}

Hausdorff matrices have played an important role in summability theory and are linked with the moment problem for a finite interval. The matrices of such standard methods of summability as the Cesàro, the Hölder, the Euler and the wighted mean methods are all Hausdorff matrices.

On one hand, such matrices are used to define different types of absolute summability such as the absolute Nörlund summability, the absolute generalized Nörlund summability, the absolute Riesz summability, the absolute generalized Cesàro summability, and the absolute Euler summability. On the other hand, by many authors such notions are employed to study an interesting topic in theory of orthogonal series the so-called their absolute summability. For example, one can see the work of Tandori [19], Leindler [8-11], Okuyama and Tsuchikura [15], Okuyama [13, 14], Szalay [17], Billard [1], Grepaqevskaya [4], Spevakov and Kudrajatsev [16]. In 2002 Okuyama [14] using generalized Nörlund means has proved two theorems which give sufficient conditions in terms of the coefficients of an orthogonal series under which it is absolute generalized Nörlund summable almost everywhere.

A generalization of a definition of absolute summability given by Borwien [2] is presented in the paper of Borwein et al in [3], called the absolute generalized Hausdorff summability. After some results proved by present author ([5-7]), which generalize some of the results of the above mentioned papers, we continue here to find 
any sufficient condition under which an orthogonal series will be absolute generalized Hausdorff summable, and this is the main purpose of this paper.

\section{NOTATIONS, NOTIONS AND KNOWN RESULTS}

Let $\sum_{n=0}^{\infty} a_{n}$ be a given infinite series with its partial sums $\left\{s_{n}\right\}$. Then, let $p$ denotes the sequence $\left\{p_{n}\right\}$ of real numbers. For two given sequences $p$ and $q$, the convolution $(p * q)_{n}$ is defined by

$$
(p * q)_{n}=\sum_{m=0}^{n} p_{m} q_{n-m}=\sum_{m=0}^{n} p_{n-m} q_{m} .
$$

When $(p * q)_{n} \neq 0$ for all $n$, the generalized Nörlund transform of the sequence $\left\{s_{n}\right\}$ is the sequence $\left\{t_{n}^{p, q}\right\}$ obtained by putting

$$
t_{n}^{p, q}=\frac{1}{(p * q)_{n}} \sum_{m=0}^{n} p_{n-m} q_{m} s_{m} .
$$

The infinite series $\sum_{n=0}^{\infty} a_{n}$ is absolutely summable $(N, p, q)$ if the series

$$
\sum_{n=1}^{\infty}\left|t_{n}^{p, q}-t_{n-1}^{p, q}\right|
$$

converges, and is written in brief

$$
\sum_{n=0}^{\infty} a_{n} \in|N, p, q| .
$$

The method $|N, p, q|$ of summability was introduced by Tanaka [18].

Let $\left\{\varphi_{n}(x)\right\}$ be an orthonormal system defined in the interval $(a, b)$. We assume that $f(x)$ belongs to $L^{2}(a, b)$ and

$$
f(x) \sim \sum_{n=0}^{\infty} c_{n} \varphi_{n}(x),
$$

where $c_{n}=\int_{a}^{b} f(x) \varphi_{n}(x) d x,(n=0,1,2, \ldots)$.

In [14] is written

$$
R_{n}:=(p * q)_{n}, R_{n}^{j}:=\sum_{m=j}^{n} p_{n-m} q_{m}
$$

where

$$
R_{n}^{n+1}=0, R_{n}^{0}=R_{n},
$$

and the following two theorems are proved: 
Theorem 1 ([14]). If the series

$$
\sum_{n=1}^{\infty}\left\{\sum_{j=1}^{n}\left(\frac{R_{n}^{j}}{R_{n}}-\frac{R_{n-1}^{j}}{R_{n-1}}\right)^{2}\left|c_{j}\right|^{2}\right\}^{\frac{1}{2}}
$$

converges, then the orthogonal series

$$
\sum_{n=0}^{\infty} c_{n} \varphi_{n}(x)
$$

is summable $|N, p, q|$ almost everywhere.

Theorem 2 ([14]). Let $\{\Omega(n)\}$ be a positive sequence such that $\{\Omega(n) / n\}$ is a non-increasing sequence and the series $\sum_{n=1}^{\infty} \frac{1}{n \Omega(n)}$ converges. Let $\left\{p_{n}\right\}$ and $\left\{q_{n}\right\}$ be non-negative. If the series $\sum_{n=1}^{\infty}\left|c_{n}\right|^{2} \Omega(n) w(n)$ converges, then the orthogonal series $\sum_{n=0}^{\infty} c_{n} \varphi_{n}(x) \in|N, p, q|$ almost everywhere, where $w(n)$ is defined by $w(j):=j^{-1} \sum_{n=j}^{\infty} n^{2}\left(\frac{R_{n}^{j}}{R_{n}}-\frac{R_{n-1}^{j}}{R_{n-1}}\right)^{2}$.

The following definition is introduced by Borwein et al in [3]. Let $M:=\left(a_{n k}\right)$ $(n, k=0,1, \ldots)$ be a matrix. For the series $\sum_{n=0}^{\infty} a_{n}$ and its partial sums $s_{n}$ let

$$
\sigma_{n}=M\left(s_{n}\right)=\sum_{k=0}^{\infty} a_{n k} s_{k}
$$

Let

$$
U_{n}=1-u_{0}+\sum_{k=0}^{n} u_{k} \quad \text { where } u_{k}>0 \text { for } k=0,1, \ldots,
$$

and assume that $\gamma$ is a real number and $\beta>0$.

We say that the series $\sum_{n=0}^{\infty} a_{n}$ is absolute summable $\left|M, u_{n}, \gamma\right|_{\beta}$ or $\sum_{n=0}^{\infty} a_{n} \in$ $\left|M, u_{n}, \gamma\right|_{\beta}$ if

$$
\sum_{n=1}^{\infty} U_{n}^{\gamma \beta+\beta-1} u_{n}^{1-\beta}\left|\sigma_{n}-\sigma_{n-1}\right|^{\beta}<\infty
$$

The main purpose of the present paper is to generalize Theorems 1 and 2 for $\left|M, u_{n}, \gamma\right|_{\beta}$ summability of the orthogonal series (2.1), where $1 \leq \beta \leq 2$. For the presentation of the main results we need to introduce some further notations and requirements. Firstly, we suppose that $M:=\left(a_{n k}\right)$ is a normal matrix, i.e. a lower triangular matrix of non-zero diagonal entries, and secondly, for it we associate another two lower matrices $\bar{M}:=\left(\bar{a}_{n k}\right)$ and $\hat{M}:=\left(\hat{a}_{n k}\right)$ as follows:

$$
\bar{a}_{n k}:=\sum_{i=k}^{n} a_{n i}, n, i=0,1,2, \ldots
$$


and

$$
\hat{a}_{00}=\bar{a}_{00}=a_{00}, \hat{a}_{n k}=\bar{a}_{n k}-\bar{a}_{n-1, k},(n=1,2, \ldots) .
$$

Throughout this paper, the letter $K$ denotes a positive constant having different values in different contexts and depending only on $k$.

The following lemma due to Beppo Levi (see, for example [12]) is often used in the theory of functions. It will need us to prove main results.

Lemma 1. If $f_{n}(t) \in L(E)$ are non-negative functions and

$$
\sum_{n=1}^{\infty} \int_{E} f_{n}(t) d t<\infty
$$

then the series

$$
\sum_{n=1}^{\infty} f_{n}(t) d t
$$

converges almost everywhere on $E$ to a function $f(t) \in L(E)$. Moreover, the series (2.3) is also convergent to $f$ in the norm of $L(E)$.

\section{NeW RESUlts}

We establish the following statement.

Theorem 3. If the series

$$
\sum_{n=1}^{\infty}\left[\left(U_{n}^{\gamma+1-\frac{1}{\beta}} u_{n}^{\frac{1}{\beta}-1}\right)^{2} \sum_{j=0}^{n}\left|\hat{a}_{n, j}\right|^{2}\left|c_{j}\right|^{2}\right]^{\frac{\beta}{2}}
$$

converges for $1 \leq \beta \leq 2$, then the orthogonal series

$$
\sum_{n=0}^{\infty} c_{n} \varphi_{n}(x)
$$

is $\left|M, u_{n}, \gamma\right|_{\beta}$-summable almost everywhere.

Proof. Let $1<\beta<2$. For the transform $\sigma_{n}(x)=M\left(s_{n}\right)(x)$ we have

$$
\begin{aligned}
\sigma_{n}(x) & =\sum_{k=0}^{n} a_{n k} s_{k}(x)=\sum_{k=0}^{n} a_{n k} \sum_{j=0}^{k} c_{j} \varphi_{j}(x) \\
& =\sum_{j=0}^{n} c_{j} \varphi_{j}(x) \sum_{k=j}^{n} a_{n k}=\sum_{j=0}^{n} \bar{a}_{n j} c_{j} \varphi_{j}(x)
\end{aligned}
$$

where $\sum_{j=0}^{k} c_{j} \varphi_{j}(x)$ is the partial sum of order $k$ of the series (2.1). 
Hence

$$
\begin{aligned}
\sigma_{n}(x)-\sigma_{n-1}(x) & =\sum_{j=0}^{n} \bar{a}_{n j} c_{j} \varphi_{j}(x)-\sum_{j=0}^{n-1} \bar{a}_{n-1, j} c_{j} \varphi_{j}(x) \\
& =\bar{a}_{n n} c_{n} \varphi_{n}(x)+\sum_{j=0}^{n-1}\left(\bar{a}_{n, j}-\bar{a}_{n-1, j}\right) c_{j} \varphi_{j}(x) \\
& =\hat{a}_{n n} c_{n} \varphi_{n}(x)+\sum_{j=0}^{n-1} \hat{a}_{n, j} c_{j} \varphi_{j}(x)=\sum_{j=0}^{n} \hat{a}_{n, j} c_{j} \varphi_{j}(x)
\end{aligned}
$$

Using the Hölder's inequality and orthogonality to the latter equality, with $r$ and $s=\frac{2}{\beta}$ such that $r+s=r s$, we obtain

$$
\begin{aligned}
\int_{a}^{b}\left|\sigma_{n}(x)-\sigma_{n-1}(x)\right|^{\beta} d x & \leq(b-a)^{1-\frac{\beta}{2}}\left(\int_{a}^{b}\left|\sigma_{n}(x)-\sigma_{n-1}(x)\right|^{2} d x\right)^{\frac{\beta}{2}} \\
& =(b-a)^{1-\frac{\beta}{2}}\left(\int_{a}^{b}\left|\sum_{j=0}^{n} \hat{a}_{n, j} c_{j} \varphi_{j}(x)\right|^{2} d x\right)^{\frac{\beta}{2}} \\
& =(b-a)^{1-\frac{\beta}{2}}\left[\sum_{j=0}^{n}\left|\hat{a}_{n, j}\right|^{2}\left|c_{j}\right|^{2}\right]^{\frac{\beta}{2}} .
\end{aligned}
$$

Thus, the series

$$
\begin{gathered}
\sum_{n=1}^{\infty} U_{n}^{\gamma \beta+\beta-1} u_{n}^{1-\beta} \int_{a}^{b}\left|\sigma_{n}(x)-\sigma_{n-1}(x)\right|^{\beta} d x \leq \\
\leq(b-a)^{1-\frac{\beta}{2}} \sum_{n=1}^{\infty}\left[\left(U_{n}^{\gamma+1-\frac{1}{\beta}} u_{n}^{\frac{1}{\beta}-1}\right)^{2} \sum_{j=0}^{n}\left|\hat{a}_{n, j}\right|^{2}\left|c_{j}\right|^{2}\right]^{\frac{\beta}{2}}
\end{gathered}
$$

converges by the assumption since the last one does. Also we note that the functions $\left|\sigma_{n}(x)-\sigma_{n-1}(x)\right|$ are non-negative and integrable, therefore according to the Lemma 1 the series

$$
\sum_{n=1}^{\infty} U_{n}^{\gamma \beta+\beta-1} u_{n}^{1-\beta}\left|\sigma_{n}(x)-\sigma_{n-1}(x)\right|^{\beta}
$$

converges almost everywhere.

For $\beta=1$ we use the Schwartz's inequality, until for $\beta=2$ we use just the orthogonality. With this the proof is completed. 
Now we shall prove a general theorem regarding to $\left|M, u_{n}, \gamma\right|_{\beta}$-summability of an orthogonal series which involves a positive sequence with certain additional conditions.

For this reason first we denote

$$
\phi^{(\beta, \gamma)}(j):=\frac{1}{j^{\frac{2}{\beta}-1}} \sum_{n=j}^{\infty}\left(n^{\frac{2}{\beta}-1} U_{n}^{\gamma+1-\frac{1}{\beta}} u_{n}^{\frac{1}{\beta}-1}\left|\hat{a}_{n, j}\right|\right)^{2}
$$

then the following theorem holds true.

Theorem 4. Let $1 \leq \beta \leq 2$ and $\{\Omega(n)\}$ be a positive sequence such that $\{\Omega(n) / n\}$ is a non-increasing sequence and the series

$$
\sum_{n=1}^{\infty} \frac{1}{n \Omega(n)}
$$

converges.

If the series

$$
\sum_{j=1}^{\infty}\left|c_{j}\right|^{2} \Omega^{\frac{2}{\beta}-1}(j) \phi^{(\beta, \gamma)}(j)
$$

converges, then

$$
\sum_{n=0}^{\infty} c_{n} \varphi_{n}(x) \in\left|M, u_{n}, \gamma\right|_{\beta}
$$

almost everywhere, where $\phi^{(\beta, \gamma)}(n)$ is defined by (3.2).

Proof. Applying the Hölder's inequality to the inequality (3.1) we get that

$$
\begin{aligned}
& \sum_{n=1}^{\infty} U_{n}^{\gamma \beta+\beta-1} u_{n}^{1-\beta} \int_{a}^{b}\left|\sigma_{n}(x)-\sigma_{n-1}(x)\right|^{\beta} d x \leq \\
& \leq K \sum_{n=1}^{\infty}\left[\left(U_{n}^{\gamma+1-\frac{1}{\beta}} u_{n}^{\frac{1}{\beta}-1}\right)^{2} \sum_{j=0}^{n}\left|\hat{a}_{n, j}\right|^{2}\left|c_{j}\right|^{2}\right]^{\frac{\beta}{2}} \\
& =K \sum_{n=1}^{\infty} \frac{1}{(n \Omega(n))^{\frac{2-\beta}{2}}}\left[(n \Omega(n))^{\frac{2}{\beta}-1}\left(U_{n}^{\gamma+1-\frac{1}{\beta}} u_{n}^{\frac{1}{\beta}-1}\right)^{2} \sum_{j=0}^{n}\left|\hat{a}_{n, j}\right|^{2}\left|c_{j}\right|^{2}\right]^{\frac{\beta}{2}} \\
& \leq K\left(\sum_{n=1}^{\infty} \frac{1}{(n \Omega(n))}\right)^{\frac{2-\beta}{2}}\left[\sum_{n=1}^{\infty}(n \Omega(n))^{\frac{2}{\beta}-1}\left(U_{n}^{\gamma+1-\frac{1}{\beta}} u_{n}^{\frac{1}{\beta}-1}\right)^{2} \sum_{j=0}^{n}\left|\hat{a}_{n, j}\right|^{2}\left|c_{j}\right|^{2}\right]^{\frac{\beta}{2}}
\end{aligned}
$$




$$
\begin{aligned}
& \leq K\left\{\sum_{j=1}^{\infty}\left|c_{j}\right|^{2} \sum_{n=j}^{\infty}(n \Omega(n))^{\frac{2}{\beta}-1}\left(U_{n}^{\gamma+1-\frac{1}{\beta}} u_{n}^{\frac{1}{\beta}-1}\right)^{2}\left|\hat{a}_{n, j}\right|^{2}\right\}^{\frac{\beta}{2}} \\
& \leq K\left\{\sum_{j=1}^{\infty}\left|c_{j}\right|^{2}\left(\frac{\Omega(j)}{j}\right)^{\frac{2}{\beta}-1} \sum_{n=j}^{\infty} n^{2\left(\frac{2}{\beta}-1\right)}\left(U_{n}^{\gamma+1-\frac{1}{\beta}} u_{n}^{\frac{1}{\beta}-1}\right)^{2}\left|\hat{a}_{n, j}\right|^{2}\right\}^{\frac{\beta}{2}} \\
& =K\left\{\sum_{j=1}^{\infty}\left|a_{j}\right|^{2} \Omega^{\frac{2}{\beta}-1}(j) \phi^{(\beta, \gamma)}(j)\right\}^{\frac{\beta}{2}},
\end{aligned}
$$

which is finite by assumption, and this completes the proof.

If $u_{n}=1$ for $n=0,1,2, \ldots$, then (2.2) is equivalent to

$$
\sum_{n=1}^{\infty} n^{\gamma \beta+\beta-1}\left|\sigma_{n}-\sigma_{n-1}\right|^{\beta}<\infty
$$

which is the defining inequality in the definition of absolute summability given by Borwein [2]. Therefore from Theorems 3-4 we immediately obtain:

Corollary 1 ([6]). If the series

$$
\sum_{n=1}^{\infty}\left[n^{2\left(\gamma+1-\frac{1}{\beta}\right)} \sum_{j=0}^{n}\left|\hat{a}_{n, j}\right|^{2}\left|c_{j}\right|^{2}\right]^{\frac{\beta}{2}}
$$

converges for $1 \leq \beta \leq 2$, then the orthogonal series

$$
\sum_{n=0}^{\infty} c_{n} \varphi_{n}(x)
$$

is $|M, \gamma|_{\beta}$-summable almost everywhere.

Corollary 2 ([6]). Let $1 \leq \beta \leq 2$ and $\{\Omega(n)\}$ be a positive sequence such that $\{\Omega(n) / n\}$ is a non-increasing sequence and the series

$$
\sum_{n=1}^{\infty} \frac{1}{n \Omega(n)}
$$

converges.

If the series

$$
\sum_{j=1}^{\infty}\left|c_{j}\right|^{2} \Omega^{\frac{2}{\beta}-1}(j) \theta^{(\beta, \gamma)}(j)
$$


converges, then

$$
\sum_{n=0}^{\infty} c_{n} \varphi_{n}(x) \in|M, \gamma|_{\beta}
$$

almost everywhere, where $\phi^{(\beta, \gamma)}(n)$ is defined by

$$
\theta^{(\beta, \gamma)}(j):=\frac{1}{j^{\frac{2}{\beta}-1}} \sum_{n=j}^{\infty}\left(n^{\gamma+\frac{1}{\beta}}\left|\hat{a}_{n, j}\right|\right)^{2} .
$$

We note that Theorems 1-2 are corollaries of the main results as well. It is enough to put in Theorems 3-4: $u_{n}=1$ for $n=0,1,2, \ldots, \gamma=0, \beta=1$, and $a_{n k}=\frac{p_{n-k} q_{k}}{R_{n}}$.

Namely, for such particular conditions we have

$$
\begin{aligned}
\hat{a}_{n, k} & =\bar{a}_{n, k}-\bar{a}_{n-1, k}=\sum_{j=k}^{n} a_{n j}-\sum_{j=k}^{n-1} a_{n-1, j} \\
& =\frac{1}{R_{n}} \sum_{j=k}^{n} p_{n-j} q_{j}-\frac{1}{R_{n-1}} \sum_{j=k}^{n-1} p_{n-1-j} q_{j}=\frac{R_{n}^{j}}{R_{n}}-\frac{R_{n-1}^{j}}{R_{n-1}} \Rightarrow \\
& \Rightarrow\left|\hat{a}_{n, k}\right|^{2}=\left(\frac{R_{n}^{j}}{R_{n}}-\frac{R_{n-1}^{j}}{R_{n-1}}\right)^{2}
\end{aligned}
$$

and similarly

$$
\phi^{(1,0)}(j):=\frac{1}{j} \sum_{n=j}^{\infty}\left(n\left(\frac{R_{n}^{j}}{R_{n}}-\frac{R_{n-1}^{j}}{R_{n-1}}\right)\right)^{2},
$$

which verify Theorems 1-2.

Remark 1. It should also noted that if we take the values of $u_{n}, \gamma, \beta$ as above and:

(i) $a_{n k}=\frac{p_{n-k}}{P_{n}}$

(ii) $a_{n k}=\frac{q_{k}}{Q_{n}}$

one can obtain the results of [15], see also corollaries proved in [14].

\section{REFERENCES}

[1] P. Billard, "Sur la sommabilité absolue des séries de fonctions orthogonales," Bull. Sci. Math., II. Sér., vol. 85, pp. 29-33, 1961.

[2] D. Borwein, "On strong ad absolute summability," Proc. Glasg. Math. Assoc., vol. 4, pp. 122-139, 1960.

[3] D. Borwein, F. P. Cass, and J. E. Sayre, “On absolute generalized Hausdorff summability,” Arch. Math., vol. 46, pp. 419-427, 1986.

[4] L. V. Grepachevskaya, "Absolute summability of orthogonal series," Mat. Sb. (N.S.), vol. 65, no. 107, pp. 370-389, 1964.

[5] X. Z. Krasniqi, "On absolute weighted mean summability of orthogonal series," Selçuk J. Appl. Math., vol. 12, no. 2, pp. 63-70, 2011. 
[6] X. Z. Krasniqi, “On $|A, \delta|_{k}$-summability of orthogonal series," Math. Bohem., vol. 137, no. 1, pp. $17-25,2012$.

[7] X. Z. Krasniqi, "On absolute almost generalized Nörlund summability of orthogonal series," Kyungpook Math. J., to appear.

[8] L. Leindler, "On the absolute Riesz summability of orthogonal series," Acta Sci. Math., vol. 46, pp. 203-209, 1983.

[9] L. Leindler, "On the newly generalized absolute Riesz summability of orthogonal series," Anal. Math., vol. 21, no. 4, pp. 285-297, 1995.

[10] L. Leindler and K. Tandori, "On absolute summability of orthogonal series," Acta Sci. Math., vol. 50, pp. 99-104, 1986.

[11] L. Leindler, "Über die absolute Summierbarkeit der Orthogonalreihen,” Acta Sci. Math., vol. 22, pp. 243-268, 1961.

[12] I. P. Natanson, Theory of functions of a real variable (2 vols). New York: Frederick Ungar, 1955, 1961.

[13] Y. Okuyama, "On the absolute Nörlund summability of orthogonal series," Proc. Japan Acad., Ser. A, vol. 54, pp. 113-118, 1978.

[14] Y. Okuyama, "On absolute generalized Nörlund summability of orthogonal series," Tamkang J. Math., vol. 33, no. 2, pp. 161-165, 2002.

[15] Y. Okuyama and T. Tsuchikura, "On the absolute Riesz summability of orthogonal series," Anal. Math., vol. 7, pp. 199-208, 1981.

[16] V. N. Spevakov and A. B. Kudrjavcev, “Absolute summability of orthogonal series by Euler's method," Math. Notes, vol. 21, pp. 29-32, 1977.

[17] I. Szalay, "On generalized absolute Cesàro summability of orthogonal series," Acta Sci. Math., vol. 32, pp. 51-57, 1971.

[18] M. Tanaka, "On generalized Nörlund methods of summability," Bull. Aust. Math. Soc., vol. 19, pp. 381-402, 1978.

[19] K. Tandori, “Über die orthogonalen Funktionen. IX.” Acta Sci. Math., vol. 21, pp. 292-299, 1960.

Author's address

Xhevat Z. Krasniqi

University of Prishtina, Department of Mathematics and Computer Sciences, Avenue Mother Theresa 5, 10000 Prishtinë, Republic of Kosova

E-mail address: xhevat.krasniqi@uni-pr.edu 